\title{
Eustachian Tube dysfunction in chronic rhinosinusitis: pre- and post-operative results following endoscopic sinus surgery, a prospective study*
}

Philippe F. D. Bowles, Satish Agrawal, Mahmoud A. Salam

ENT Department, Ipswich Hospital NHS Trust, Ipswich, United Kingdom
Rhinology 57: 1, 73 - 77, 2019

https://doi.org/10.4193/Rhin18.208

*Received for publication:

September 3, 2018

Accepted: October 26, 2018

Background: Prospective study investigating the incidence of concurrent Eustachian Tube dysfunction (ETD) in patients with CRS refractory to medical therapy, and the effect of Endoscopic Sinus Surgery (ESS) on ETD in this patient group.

Methods: Prospective study of 57 CRS patients. Outcome measures were SNOT-22 and ETDQ-7 questionnaires, tympanometry and Valsalva manoeuvre recorded pre-operatively and at 3 and 9 months post ESS.

Results: There was a moderate positive correlation between pre-operative ETDQ-7 and SNOT 22 scores $(r=0.5715, p<0.0001) .68 \%$ of patients recorded positive ETDQ-7 scores pre-operatively, mean=20.6 (SD \pm 10.34$)$. Mean ETDQ-7 scores were significantly lower at 3 months; mean=11.4 (SD \pm 5.65$)(P<0.0001)$ and 9 months mean=11.4 (SD \pm 6.15$)(P<0.0001)$ following ESS. Type A tympanograms increased form $76.6 \%$ pre-operatively, to $94.5 \%$ at 3 months and $96 \%$ at 9 months. Reported positive Valsalva increased from $38 \%$ pre-operatively to $96 \%$ at 3 and 9 months. Mean ETDQ-7 scores were higher in the CRSwNP group; 24.34 (SD \pm 9.2 ) compared to the CRSsNP group; 18.11 (SD \pm 10.3$)$, $(\mathrm{p}=0.6101) .16$ patients in the cohort had existing diagnoses of asthma, of which 4 had documented aspirin sensitivity. The mean pre-operative SNOT-22 score in this overall subgroup was 64.81 (SD= \pm 20.13 ) compared with 49.07 ( $S D= \pm 21.37)$ in non-asthmatic patients $(p=0.0168)$.

Conclusions: We found a high incidence of concurrent ETD symptoms in patients with severe CRS, which improve following ESS. Further research is required to better understand the association between CRS and ETD in order to provide effective treatments.

Key words: sinusitis, Eustachian tube, endoscopic surgical procedure, auditory tube, pharyngotympanic tube.

\section{Introduction}

Eustachian tube dysfunction (ETD) has been estimated to affect $1 \%$ of the UK population, while ETD and related pathologies (tympanic membrane retraction and otitis media with effusion) account for over 2 million adult outpatient clinic appointments in the US annually ${ }^{(1,2)}$. The Eustachian tube provides middle ear ventilation and pressure equalization, as well as mucocillary clearance and protection of the middle ear from sounds and nasopharyngeal pathogens ${ }^{(3)}$. Anatomical obstruction of the Eustachian tube resulting from inflammatory conditions of the nasopharynx may lead to dilatory ETD, with smoking, allergic rhinitis, chronic rhinosinusitis and laryngopharyngeal reflux all having been proposed as potential contributing factors affecting tubal function ${ }^{(4-8)}$. Several recent studies have reported a high incidence of concurrent ETD in CRS patients and more widely, in those patients presenting to rhinology clinics ${ }^{(9-13)}$. The Sino-nasal outcome test (SNOT-22) is a validated patient reported questionnaire validated for CRS and contains two questions related to symptoms of ETD (Qu. 7 'ear fullness' and Qu. 9'ear pain/pressure'). We observed that CRS patients presenting to our rhinology clinic with severe CRS also often reported symptoms suggestive of ETD, and that these symptoms became 
less troublesome following Endoscopic Sinus Surgery (ESS). The purpose of this study was to prospectively investigate the incidence of ETD symptoms in a consecutive series of patients with CRS refractory to medical management, before and after ESS.

\section{Material and Methods}

This was a prospective study of 57 (F:20 , M:37, age: 24 - 89yrs) consecutive patients undergoing ESS for CRSwNP or CRSsNP refractory to full medical therapy (2012 EPOS guidelines) ${ }^{(8)}$. Data collection was between 31.08.2016 and 30.11.2017. Preoperative assessment included history and examination, flexible nasendoscopy (FNE), CT-Scan (nose and paranasal sinuses) and completion of SNOT-22 questionnaires. In addition, patients completed a Eustachian Tube Dysfunction 7 (ETDQ-7) questionnaire, and underwent tympanometry. Tympanometry was performed by the department Senior Clinical Audiologist. Tympanometric data were categorized into three groups using the Jerger classification; type $\mathrm{A}$ (peak at $0 \mathrm{~mm} \mathrm{H}_{2} \mathrm{O}$, range between $-100-+200 \mathrm{~mm} \mathrm{H}_{2} \mathrm{O}$ ), type $B$ (flat, or very low, rounded peak with normal ear canal volume), \& type $C$ (peak in negative pressure region between - $\left.100--600 \mathrm{~mm} \mathrm{H}_{2} \mathrm{O}\right){ }^{(9,10)}$. Patient reported ability to perform an effective Valsalva manoeuvre was also recorded pre-operatively. The outcome measures were then collected at 3 months and 9 months post ESS.

\section{Patient selection}

Inclusion criteria were patients with persisting symptoms of CRSsNP and CRSwNP with radiological evidence of sinus disease on nasendoscopy and CT scanning despite a minimum of 6 months of medical therapy (all patients received saline nasal douching, topical and oral steroids and at least one course of oral antibiotics). Exclusion criteria were patients with pre-existing otological disease, patients responding to medical therapy, patient preference for continuing medical therapy over surgical intervention and patients with severe systemic disease preventing general anaesthetic.

\section{Intervention}

All procedures were performed under a general anaesthetic, with topical nasal decongestant applied in the anaesthetic room. A standard surgical approach was used by a single operating surgeon guided by radiological, pre-operative FNE and intra-operative findings. All patients underwent uncinectomy, middle meatal antrostomy and anterior and posterior ethmoidectomy. Additionally, sphenoidotomy and clearance of the frontal recess were undertaken where indicated by disease extent. Patients were advised to continue saline nasal douching and topical steroid applications post-operatively.

\section{Statistical analysis}

Statistical analysis was performed using GraphPad Prism (ver-

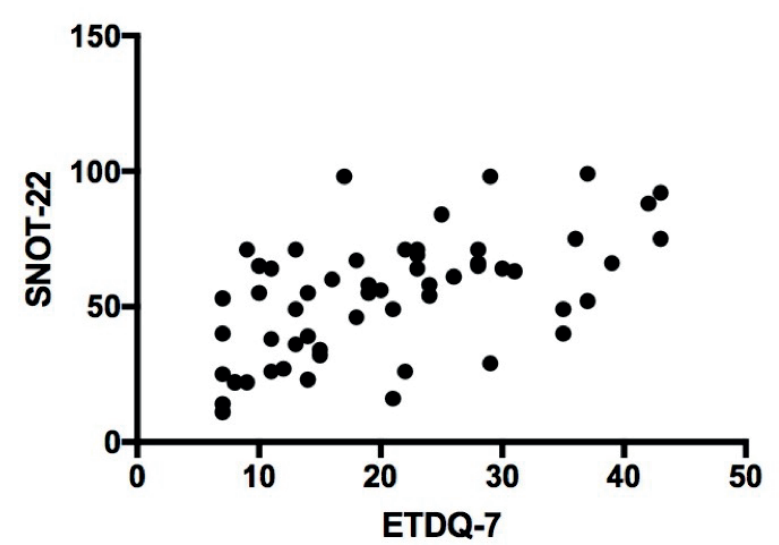

Figure 1. Correlation between ETDQ-7 and SNOT 22 scores.

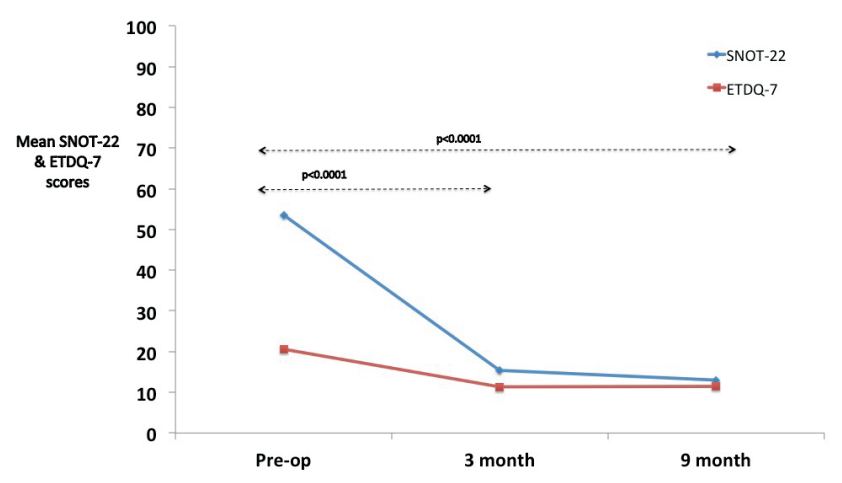

Figure 2. SNOT-22 and ETDQ-7 scores pre-operatively, and at 3 and 9 months post endoscopic sinus surgery.

sion 6.00 for MacOS, GraphPad Software, La Jolla, San Diego, CA, USA; www.graphpad.com). Shapiro-Wilk normality test was applied to all group scores and histograms compared. Gaussian distribution was not found to be unanimous across all groups and non-parametric analysis was therefore applied (significance was set at $\mathrm{p} \leq 0.05$ ). Spearman Rank correlation co-efficient analysis was applied to assess the correlation between SNOT-22 and ETDQ-7 scores.

\section{Ethical considerations}

This study was registered with the hospital Clinical Governance department and approved locally (audit section, study no. 5392). All patients participating in the study were supplied with patient information sheets and underwent informed consent in the outpatient setting prior to the procedure. Informed consent was confirmed on the day of surgery.

\section{Results}

There was a moderate positive correlation between pre-operative ETDQ-7 and SNOT 22 scores (Spearman rank correlation co-efficient; $r=0.57, \mathrm{Cl} 0.36-0.73, \mathrm{n}=57, \mathrm{p}<0.0001$ ) (Figure 1). 68\% $(n=39)$ of patients recorded positive ETDQ-7 scores (total score $>$ 13.5) pre-operatively suggestive of ETD, mean 20.6 (SD \pm 10.34 ). 


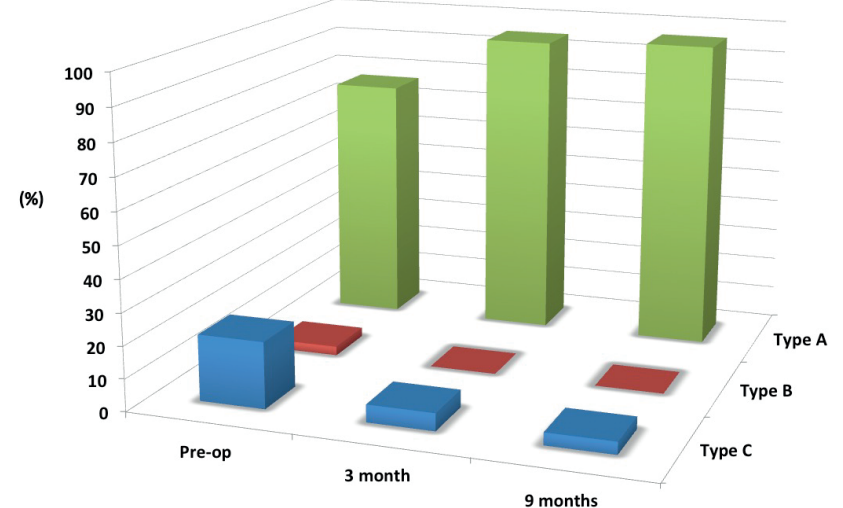

Figure 3. Tympanography pre-operatively and at 3 months and 9 months post ESS.

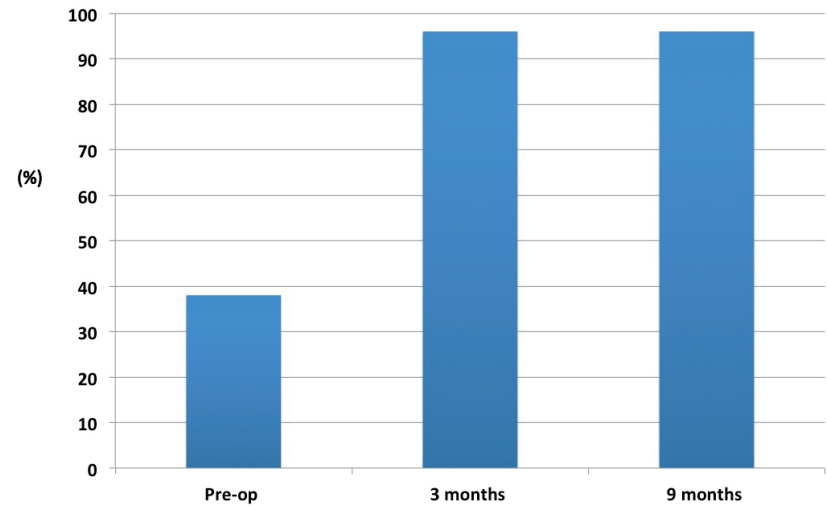

Figure 4. Percentage of patients reporting ability to perform an effective Valsalva manoeuvre pre-operatively and at 3 months and 9 months post ESS.
Mean ETDQ-7 scores were significantly lower at 3 month follow up; $11.4(S D \pm 5.65)(P<0.0001)$ [91\% follow up, $n=51]$, and 9 months $11.4(S D \pm 6.15)(P<0.0001)$ [38\% follow up $n=26]$ following ESS (Figure 2). Type A tympanograms increased from $76.6 \%$ ( $n=114$ ears) pre-operatively, to $94.5 \%(n=92)$ at 3 months and $96 \%(n=52)$ at 9 months (Figure 3). Patients able to perform an effective Valsalva increased from $38 \%$ pre-operatively to $96 \%$ at 3 and 9 months (Figure 4). 23\% ( $n=6)$ of patients recorded positive ETDQ-7 scores at 9 month follow up. Mean ETDQ-7 scores were higher in the CRSwNP group ( $n=23) ; 24.34$ (SD \pm 9.2 ) compared to the CRSsNP group ( $n=34) ; 18.11$ (SD \pm 10.3 ), although this did not reach significance $(p=0.6101)$. Pre-operative tympanometry in the CRSwNP group showed $72.06 \%$ ( $n=49$ ears) to have type A tympanograms, compared with $71.74 \%$ ( $n=33$ ears) in the CRSsNP group. Post-operative SNOT-22 scores were significantly lower at 3 and 9-month follow up in both groups $(p<0.0001) .16$ patients in the cohort had existing diagnoses of asthma, of which 4 had documented aspirin sensitivity. The mean pre-operative SNOT-22 score in this overall subgroup was 64.81 ( $S D= \pm 20.13$ ) compared with 49.07 ( $S D= \pm 21.37$ ) in nonasthmatic patients $(p=0.0168)$. The mean pre-operative SNOT-22 score in the subgroup with asthma and aspirin sensitivity $(n=4)$ was $81.75(S D= \pm 20.13)(p=0.0199)$. The mean ETDQ-7 score in the overall subgroup of patients with asthma was 22.62 (SD $\pm 9.47)$ and 29.5 ( $S D= \pm 9.47$ ) in the group of patients with asthma and aspirin sensitivity, compared with $19.85(\mathrm{SD}= \pm 10.55)$ in patients without asthma ( $p=0.3568 \& p=0.2744$ respectively).

\section{Discussion}

Synopsis of key findings

Our findings showed a high incidence of ETD (68\%) in a patient cohort with severe CRS. There was a moderate positive correlation between SNOT-22 scores and ETDQ-7 scores. ETDQ-7 scores were significantly reduced at 3 months and 9 months following
ESS suggesting a reduction in ETD in CRS patients undergoing ESS. $23 \%$ of patients recorded ETDQ-7 scores $>13.5$ at 9 month follow up. There was a reduction in negative pressure tympanograms post ESS, and an increase in patient reported ability to perform an effective Valsalva manoeuvre. CRSwNP were found to have a higher mean ETDQ-7 score compared to CRSsNP, although tympanometry in both CRSwNP and CRSsNP groups were similar. Asthmatic patients had statistically significant higher pre-operative SNOT-22 scores than non-asthmatic patients, as well as higher ETDQ-7 scores although this did not reach significance.

\section{Comparisons with other studies}

While there is relative paucity of prospective studies employing disease specific outcome measures for ETD in rhinology patients, several researchers have investigated the association between ETD and CRS ${ }^{(6,7,11,12)}$. In a recent retrospective study, Maniakas et al. assessed the incidence of ETD in a CRS patient population and the response to FESS ${ }^{(13)}$. By pooling data from 5 previously conducted CRS studies (131 patients) and using questions in the SNOT-22 pertaining to ear symptoms (questions 7 \& 9) as a marker of ETD symptoms, the researchers found a greater incidence of reported ear symptoms in the CRS group when compared with a control group with $60.1 \%$ reporting 'ear fullness' and 29.8\% 'ear pain/discomfort'. Following FESS, 78.8\% of patients recorded an improvement in 'ear fullness' scores ( $82.5 \%$ follow up) and $73.3 \%$ an improvement in 'ear pain/discomfort' scores (76.9\% follow up) after a mean follow up period of 3.8 months. The authors conclude that symptoms suggestive of ETD are frequent in patients with CRS who fail maximal medical therapy and that the symptoms fall to a level comparable to a control group following FESS. The study did not find a higher incidence or 'ear symptoms' in the CRSwNP subgroup ${ }^{(13)}$. A prospective study of 492 patients referred to a specialist rhi- 
nology clinic found that $43.3 \%(n=213)$ recorded mean ETDQ-7 scores suggestive of ETD (>2.1). A strong correlation was found between SNOT-22 scores and ETDQ-7 scores, and a very correlation between ETDQ-7 scores and the SNOT-22 ear subdomain scores. The authors conclude that there is an increased prevalence of otological symptoms and ETD in patients presenting with rhinology symptoms ${ }^{(7)}$. The lower incidence of ETD in this patient group than that found in our own study may be in part explained by the inclusion of a broader group of rhinology patients than CRS patients alone, with the allergic fungal sinusitis sub-group found to have significantly lower ETDQ-7 scores than patients with other forms of rhinological disease ${ }^{(7)}$. Stoikes et al. assessed the effects of ESS on ETD in 168 consecutive CRS patients who had previously undergone ESS following failure of medical management ${ }^{(11)}$. Participants were asked to retrospectively complete a questionnaire recording symptoms of; "ear fullness and congestion", "ear crackling and popping", "dizziness" and "ear pain" before and after ESS. Ear symptoms were reported in $15-42 \%$ (with $42 \%$ experiencing symptoms of ear fullness). By applying the outcome measure retrospectively, a significant treatment effect was found in all otological symptoms following ESS, leading the authors to conclude that symptoms of tubal dysfunction occur commonly in patients with severe CRS and that these symptoms appear to improve with ESS ${ }^{(11)}$. The retrospective study design implies risk of recall bias. A further study assessed SNOT-22 and ETDQ-7 scores in 119 patients attending a rhinology clinic and found a significantly higher ETDQ-7 score in rhinology patients than in the control group. The researchers also found within the rhinology population, that ETD symptoms were more prevalent in CRSwNP patients. The authors conclude that ETD is commoner in rhinology patients than in the general population ${ }^{(12)}$.

\section{Clinical applicability}

This current study offers a prospective investigation into the incidence of ETD in CRS patients and it's evolution following ESS using both CRS and ETD specific outcome measures. The high prevalence of ETD in this patient group is comparable to that reported in other studies and supports the assertion that ear symptoms are under appreciated in CRS patients ${ }^{(7,13)}$. The significant improvement in ETDQ-7 scores, tympanometry and patient reported Valsalva manoeuvres following ESS suggest an improvement in ETD following ESS in patients with severe CRS. These findings also support the suggestion that CRS may affect tubal function and that in patients presenting with ETD symptoms, assessment and management of concurrent CRS should be considered ${ }^{(4,11)}$. We found that in the majority of cases ETD symptoms improved or resolved following ESS. However, ETD symptoms did persist in a subgroup with $23 \%$ of patients found to have positive ETDQ-7 scores at 9 months following ESS.. Further research is required to better understand the aeti- ology and impact of ETD in CRS patients, and provide effective treatments. Early studies assessing emerging therapies aimed at treating ETD, such as balloon dilation of the Eustachian Tube (tuboplasty), have shown encouraging results and may represent a potential therapeutic option in this patient group ${ }^{(14-17)}$. However, they have yet to be validated as a therapeutic option by high quality randomized controlled studies.

\section{Strengths and limitations}

A gold standard diagnostic test and outcome measure for ETD remains lacking ${ }^{(3,16)}$. However, the ETDQ-7 questionnaire has been validated for the assessment of ETD with $100 \%$ sensitivity and specificity reported ${ }^{(6,18)}$. It has been widely applied as an outcome measure for ETD ${ }^{(6,16,19)}$. While this study reports higher overall follow up rates and duration than previous studies, a nine-month follow up rate of $38 \%$ is a limitation of this study. Contributing factors to this low follow up rate are resource constraints on outpatient clinics, and patient perception of the benefits of attending more than one follow up appointment following symptom resolution. While it might be argued that this patient cohort could be considered as it's own control group having been followed up through the process of attempted medical therapy, that there is no true control group is a further limitation of the study.

\section{Conclusions}

ETD symptoms are an important factor in CRS patients. Our study results suggest that there is a high incidence of concurrent ETD symptoms in patients with severe CRS, and that these symptoms improve following ESS. Further research is required to better understand the association between CRS and ETD and it's impact on patients in order to provide effective treatments. Patients with persisting ear symptoms following ESS may represent a suitable patient group for treatment with emerging therapies directed at the Eustachian tube such as balloon tuboplasty.

\section{Key points}

- A high proportion of patients with severe CRS experience concurrent ETD symptoms .

- $\quad$ ETD symptoms in patients with severe CRS improve following ESS.

- ETD symptoms are an important, and often overlooked, factor in this patient group.

- Patients with persisting ETD symptoms following ESS may constitute a suitable group for consideration of treatment with balloon Eustachian tuboplasty.

- Identification and management of concurrent nasopharyngeal inflammatory conditions should be considered in patients presenting with ETD symptoms. 


\section{Acknowledgements}

The authors extend their sincere appreciation to Pamela Bradley at the Ipswich Hospital Audit department for her thorough and careful data management and to Janet Bayliss and Kathy Moll at Ipswich Hospital Library for their expert assistance.

\section{Authorship contribution}

All authors have contributed to the study design, data collection, analysis, and manuscript preparation for this study.

\section{Conflict of interest}

None to declare

\section{References}

1. Browning G, Gatehouse S. The prevalence of middle ear disease in the adult British poulation. Clin Otolaryngol. 1992;17(4):31721.

2. Vila PM, Thomas T, Liu C, Poe D, Shin JJ. The Burden and Epidemiology of Eustachian Tube Dysfunction in Adults. In: Otolaryngology - Head and Neck Surgery. 2017;156(2): p. 278-84.

3. Schilder AGM, Bhutta MF, Butler CC, et al. Eustachian tube dysfunction: Consensus statement on definition, types, clinical presentation and diagnosis. Clin Otolaryngol. 2015:40(5):407-11.

4. Kivekas I, Chao WC, Faquin W, et al Histopathology of balloon-dilation eustachian tuboplasty. Laryngoscope. 2015;125(2):436-41.

5. Buchman CA, Doyle WJ, Swarts JD. Effects of Nasal Obstruction on Eustachian Tube Function and Middle Ear Pressure. Acta Otolaryngol. 1999; 119(3): 351-5.

6. Tangbumrungtham N, Patel VS, Thamboo A, et al. The prevalence of Eustachian tube dysfunction symptoms in patients with chronic rhinosinusitis. Int Forum Allergy Rhinol. 2018; 8(5): 620-623.

7. Marino $M$, Ling L, Yao W, Luong A, MJ C Eustachian tube dysfunction symptoms in patietnts treated in a tertiary rhinology clinic. Int Forum Allergy Rhinol. 2017;7(12):1135-9.

8. Fokkens WJ, Lund V, Mullol J, Bachert C, Alobid I, Baroody F. EPOS European Position
Paper on Rhinosinusitus and Nasal Polyps 2012. Rhinology. 2012; 23: 1-298.

9. Warner G, Burgess A, Patel S, MartinezDevesa P, Corbridge R. Otolaryngology and Head and Neck surgery. New York: Oxford University Press; 2009. 298-299.

10. Jerger J. Clinical experience with impedance audiometry. Arch Otolaryngol - Head Neck Surg. 1970;4:311-24

11. Stoikes NFN, Dutton JM. The Effect of Endoscopic Sinus Surgery on Symptoms of Eustachian Tube Dysfunction. Am J Rhinol. 2005; 19(2): 199-202.

12. Rennie CE, Gutierrez M, Darby Y, Lund VJ. Investigation of the incidence of Eustachian tube dysfunction in patients with sinonasal disease. Rhinol Online. 2018;1:85-9.

13. Maniakas A, Desrosiers M, Asmar M, et al. Eustachian tube symptoms are frequent in chronic rhinosinusitis and respond well to endoscopic sinus surgery. Rhinology. 2018;56(2):118-21.

14. Schroder S, Lehmann M, Sudhoff $H$, Ebmeyer J. Assessment of chronic obstructive eustachian tube dysfunction: Evaluation of the German version of the Eustachian Tube Dysfunction Questionnaire. HNO. 2014;62(160):162-4.

15. Ockermann T, Reineke U, Upile T, Ebmeyer J, Sudhoff HH. Balloon dilatation Eustachian Tuboplasty: A clinical study. Laryngoscope. 2010;120(7):1411-6.

16. Bowles PF, Agrawal S, Salam MA. Balloon tuboplasty in patients with Eustachian tube dysfunction: a prospective study in
39 patients (55 ears). Clin Otolaryngol. 2017;42(5):1057-1060

17. Silvola J, Kivekäs I, Poe D. Balloon dilation of the cartilaginous portion of the Eustachian tube. Otolaryngol - Head Neck Surg. 2014;151(1):125-30

18. McCoul ED, Anand VK, Christos PJ Validating the clinical assessment of eustachian tube dysfunction: The eustachian tube dysfunction questionnaire (ETDQ-7). Laryngoscope. 2012;122(5):1137-41.

19. Mccoul ED, Anand VK. Eustachian tube balIoon dilation surgery. Int Forum Allergy Rhinol. 2012;2(3):191-8.

Philippe F. D. Bowles

ENT Department

Ipswich Hospital NHS Trust

Heath Rd.

Ipswich IP4 5PD

United Kingdom

Tel: +44 1473712233

E-mail: philippe.bowles@nhs.net 